\title{
El paradigma de la complejidad en discursos sobre formación docente en ciencias
}

\author{
The paradigm of complexity in discourses \\ on science teachers' formation
}

María Virginia Luna ${ }^{1}$

\begin{abstract}
Resumen: Este trabajo analiza un corpus de discursos contemporáneos sobre formación de profesores en Ciencias, principalmente de procedencia argentina, que refieren a la incorporación de planteamientos del Paradigma de la Complejidad como criterio para innovar contenidos y métodos en los Profesorados. El análisis arrojó que en los documentos, tanto los diagnósticos sobre el estado de los conocimientos de la formación como las transformaciones que promueven, omiten la situacionalidad de las instituciones formadoras y las lógicas epistemológicas que históricamente han conformado sus currículos. Esto lleva a concluir que, contrariamente a la retórica y objetivos que persiguen, los documentos reeditan aspectos de una epistemología clásica. A partir de allí avanzamos hacia una noción de formación docente que enfatiza su carácter de espacio de producción de conocimientos en vez de concebirla como mera instancia reproductora de los discursos del campo científico-académico. Para ello recuperamos el concepto de dispositivo pedagógico de Basil Bernstein.
\end{abstract}

Palabras clave: Formación docente. Enseñanza de las ciencias. Paradigma de la complejidad. Basil Bernstein.

\begin{abstract}
This work analyzes a corpus of contemporary discourses on science teachers' formation and refers to the incorporation of the Paradigm of Complexity as criterion to introduce new contents and methods into Professorships. The results of the analysis show that in the documents, the diagnoses on state-of-the-art knowledge as well as the promoted transformations, omit the institutional context and the epistemological logic that historically have shaped its curricula. Thus it leads to concluding that, contrary to the rhetoric and aims that the documents aspire to, they re-edit aspects of a classic epistemology. We suggest the emphasizing of teacher formation as a space for the production of knowledge instead of conceiving it as mere reproduction of academic scientific discourses. For it we use Basil Bernstein's concept of pedagogic device.
\end{abstract}

Keywords: Teachers education. Science education. Paradigm of complexity. Basil Bernstein.

\footnotetext{
${ }^{1}$ Facultad de Ciencias de la Educación, Universidad Nacional de Entre Ríos, Laprida 1856, Esperanza, C. P. 3080 , Provincia de Santa Fe, Argentina. Email: <virginialuna11@gmail.com>
} 


\section{Introducción}

Aspectos de las últimas reformas político-curriculares de la formación docente (FD) en Argentina han manifestado la necesidad de mejorar su calidad a partir de la inclusión de perspectivas de la Ciencia y la Tecnología orientadas hacia el paradigma de la Complejidad, con el objetivo de "actualizar" la enseñanza ${ }^{2}$. Este trabajo se centra en analizar algunos discursos contemporáneos sobre formación de profesores de Ciencias que refieren a la incorporación de recientes planteamientos vinculados con la Complejidad como criterio para innovar contenidos y métodos en los Profesorados. Se enmarca en un trabajo de tesis doctoral que busca historizar y caracterizar los discursos institucionales y estatales sobre la Formación Docente en Física en las últimas décadas en Argentina.

En momentos como los actuales la formación docente en Argentina vuelve a ser objeto de debates y propuestas de transformación en el ámbito de las políticas públicas. Tanto la Ley Nacional de Educación, como la institucionalización de organismos estatales dedicados a políticas de FD y la elaboración de proyectos nacionales de mejoramiento de la educación en ciencias, plantean los nuevos marcos en los que se asienta el debate sobre la formación de los profesores. De este modo y, especialmente, la Formación Docente en Ciencias ha sido un punto saliente de la agenda educativa oficial. Por todo ello, cobra especial relevancia indagar algunos aspectos y características de los planteos que aparecen en discursos sobre Formación Docente en relación al Paradigma de la Complejidad y las posibilidades de innovación de la enseñanza.

Como referentes empíricos se tomaron los últimos documentos emanados del Estado Nacional Argentino para las políticas de Formación Docente, planes y programas vigentes de Profesorados en Física de Argentina y artículos de Didáctica de las Ciencias Naturales. En líneas generales, se ha encontrado que los documentos se ordenan discursivamente por medio de una lógica "diagnóstico-solución” y de una interpretación dicotómica de las prácticas de enseñanza, calificadas éstas como "tradicionales" o "innovadoras", lo que origina, paradójicamente, la reedición de aspectos de una epistemología clásica, tal como se mostrará más adelante.

\section{Delimitación de la perspectiva de análisis. Metodología}

Este trabajo ha procedido de un recorte que intentó focalizar las reflexiones en los aspectos que relacionan específicamente el mejoramiento de la formación de profesores con la renovación de la enseñanza de las ciencias desde los aportes del denominado Paradigma de la Complejidad. En este sentido, se analizaron discursivamente documentos sobre Formación Docente en Ciencias en Argentina, de procedencia oficial e institucional (diseños curriculares, lineamientos curriculares, recomendaciones de comisiones de expertos, planes de estudio, programas de cátedras) y algunos artículos de producción académica provenientes del área de la Didáctica de las Ciencias Naturales. El corpus es acotado y fue intencionadamente conformado, pues su construcción respondió al interés de analizar enunciados representativos de unas

\footnotetext{
${ }^{2}$ Se puede consultar, por ejemplo: <http://www.me.gov.ar/doc_pdf/doc_comision.pdf $>$. Visitado el: 23 jul. 2014. 
nociones con resonancia actual en la educación científica y en la formación de profesores de Ciencias Naturales.

El análisis se organizó con base a los siguientes interrogantes: ¿qué se demanda a la FD cuando se apela a la "Complejidad" como noción clave de los cambios?, ¿qué problemas se visualizan como prioritarios de resolver en la formación?, ¿̇a qué sentidos se anudan las ideas de "enseñanza tradicional", "ciencia clásica" y sus significados oponentes?, ¿qué significados admite la Complejidad como noción fuerte de las nuevas propuestas de formación?, ¿cómo se despliega discursivamente el juego de oposición entre visiones tradicionales e innovadoras de la enseñanza y la formación?, ¿qué efectos epistemológicos se producen en la organización de las propuestas de enseñanza a futuros profesores?

En la lectura del corpus no se examinó el ajuste de los documentos a lo que se produce en el campo científico o epistemológico respecto de la Complejidad para evaluar allí aproximaciones entre unos discursos y otros. Lo que se intentó, más bien, fue describir y comprender, por ejemplo, cómo aparece referenciada la Complejidad, cómo se enuncian allí las cuestiones epistemológicas, en qué prácticas curriculares o disposiciones de saberes concretas se instalan las revisiones de paradigmas.

Todo discurso, en este caso sobre formación de profesores de ciencias, es producido en medio de relaciones de poder donde se intentan fijar o transformar sentidos. Por ende, todo discurso es productor de realidad y no reflejo de ella. A partir de esto se buscó analizar las articulaciones de sentido pretendidas y los significados excluidos en la relación que los propios discursos establecen entre el paradigma de la Complejidad y la innovación de la enseñanza a futuros docentes.

Estos planteos se asientan sobre una crítica a la epistemología clásica que admitía que los discursos y los saberes eran el producto de la relación directa entre un sujeto cognoscente y un objeto conocido. Especialmente recuperamos esta crítica de los trabajos arqueológicos de Michel Foucault (1972), en los que muestra que detrás de los discursos que denominamos ciencias o disciplinas no es posible hallar una correspondencia entre un sujeto que se "propone" conocer un "objeto" y "va en busca" de él. Existen prácticas discursivas al interior de distintas prácticas sociales que operan creando y transformando los objetos de los que habla un discurso. Este autor muestra, por ejemplo, de qué manera el objeto "locura" históricamente fue construido de manera diferente según perteneciera a las prácticas discursivas que instalan la medicina, la jurisprudencia o la psicopatología.

En términos generales, analizamos cómo documentos curriculares, producciones académicas y recomendaciones de expertos hacen jugar la noción de "Complejidad" convirtiéndola en un criterio de recontextualización (BERNSTEIN, 1998) de los saberes y prácticas pedagógicas de los profesorados. Específicamente nos atenemos a desentrañar cómo dicha noción es usada e incluida en esos planteos. Luego, veremos de qué modo algunos planes de estudio y programas de cátedra de Profesorados de Física, sobre todo aquellos que cuentan con una implementación reciente, convocan a los espacios curriculares Epistemología y Didáctica Específica como vías para introducir los planteos de la Complejidad en las propuestas de formación. 


\section{Breve panorama de replanteos epistemológicos en educación}

A partir de las décadas de 1980 y 1990 asistimos a un replanteo generalizado en los ámbitos de la producción académica y de los organismos estatales que instó a rever los postulados epistemológicos y metodológicos sobre los que la enseñanza de las ciencias y la FD se habían asentado hasta el momento. En este mismo período se desplegó también un movimiento de reformas estatales y educativas a nivel internacional, que respondió -básicamente - a las transformaciones acaecidas en el sistema capitalista mundial, con la consecuente consolidación de políticas públicas con perfil neoconservador. (POPKEWITZ, 1994).

Las revisiones se generalizaron en el campo educativo desde 1980 y algunas de ellas estuvieron estrechamente ligadas a las reformas de los sistemas de educación pública. Se abrieron líneas teóricas de reflexión particularmente en el campo de la FD y de la didáctica de la ciencia que bregaron por la instalación de nuevos modos de entender la educación científica y la enseñanza de las ciencias.

Este proceso corrió posteriormente a un vasto movimiento crítico dentro de la epistemología y del campo de la producción académica que reclamaba revisar los modelos de comprensión científica de la realidad heredados por la ciencia clásica. Dichos modelos venían poniéndose en cuestión entrado el siglo XX en disciplinas como la Física, la Físico-química, la Biología, la Matemática, la Cibernética, entre otras. Los nuevos planteos científicos y epistemológicos cuestionaron los fundamentos del conocimiento concebidos en la Modernidad, mostrando, por ejemplo el papel central del lenguaje como construcción simbólica de lo real y no como referencia transparente del mundo, la existencia del desorden y la incertidumbre como parte del "orden" de la naturaleza, el surgimiento de nuevos enfoques experimentales basados en la simulación, el desplazamiento de los campos conceptuales que rompió las barreras disciplinarias dando lugar a nuevos campos como el de las Ciencias Cognitivas o las Ciencias de la Complejidad (NAJMANOVICH, 1995, 2008), entre otras cuestiones.

Sin pretender establecer relaciones causales, podemos pensar que esos diversos procesos de revisión fueron reinterpretados e incorporados en una buena parte de los cambios impulsados en la educación en ciencias y en la formación de docentes, forjando la instalación de una nueva demanda, sobre todo hacia esta última: la de formar a los profesores de ciencias "en el paradigma de la complejidad" y así superar las obsoletas pero persistentes tradiciones de enseñanza que hacían percibir a la ciencia como un saber acabado, cerrado, certero y absoluto, reforzado esto por metodologías ancladas en la clase teórica - expositiva.

En este sentido, una nueva retórica ingresó a las dependencias pedagógicas de ministerios y a las instituciones educativas, al tiempo que fue tomada por nuevos estudios de Didáctica de la Ciencia para proponer otros modelos de enseñanza. Comenzaron a difundirse en el campo pedagógico y didáctico términos como "paradigma de la complejidad en la educación en ciencias", "el azar y la incertidumbre" para caracterizar la actividad científica en el aula, "visión más abierta, conjetural y provisoria del conocimiento científico" en planes y programas de estudio, "importancia de los procesos de producción del conocimiento" en los métodos de enseñanza, "relevancia de la historia de la ciencia" para la compresión de los contenidos de ciencias. Las últimas reformas político-curriculares de la FD en Argentina han manifestado en sus múltiples discursos, entre otras cuestiones, la necesidad de mejorar la calidad de la formación del profesorado a partir de la inclusión de perspectivas más complejas de la ciencia y la tecnología con 
el objetivo de renovar las tradicionales formas de enseñanza asentadas en paradigmas clásicos de la producción científica.

\section{La epistemología de la ciencia en discursos pedagógicos}

Desde diversos ámbitos de educación en ciencias y FD (lineamientos curriculares, informes de comisiones de expertos e investigaciones en Didáctica de las Ciencias Naturales) existe un consenso acerca de la necesidad de superar las posiciones y prácticas de transmisión de la ciencia que serían reduccionistas, fragmentadas y deterministas, para consolidar así recorridos formativos ordenados en torno a una interpretación compleja de la ciencia y de sus resultados. Recurrentemente aparece la referencia a concepciones distorsionadas de ciencia que sería imperioso transformar en los currículos de las escuelas y de los profesorados. A continuación se describen características específicas que adquieren estos planteos en los documentos analizados.

En primer lugar, la descripción de algunas condiciones del mundo contemporáneo, donde se destacan las transformaciones acaecidas en los modos de producción de información y conocimiento ligados a los procesos de globalización económica y tecnológica, opera como apertura de la mayoría de los documentos y artículos que proponen revisar las líneas epistemológicas de la educación y de la FD en ciencias. A modo de muestra de un conjunto de enunciados reiterados en trabajos actuales de Didáctica de la Ciencia que tratan la temática, citamos un fragmento, cuyo autor afirma:

Los ejes de planeación curricular, los planes de estudio, metodologías para la formación del profesor en ciencias, así como las estrategias pedagógicas y didácticas, deben estar en función del panorama complejo, que no permite la formación de un pensamiento delimitado por un solo campo disciplinar, ni mucho menos un enfoque miope en la enseñanza de la ciencia y sus posibilidades de aprendizaje en los contextos cultural, político, tecnológico, social y económico. [...] El paradigma de la complejidad empieza a sembrar sus semillas que más adelante justifican su ser - su existencia - y hacer -comportamiento- dado el avance del conocimiento, el desarrollo de la tecnología, de las comunicaciones, que expresan la globalización de los mercados, la educación, la política y la economía. (ACOSTA, 2009, p. 150)

Las "exigencias" de la denominada sociedad de la información suelen vincularse discursivamente a las nociones de complejidad, interdisciplinariedad, multidisciplinariedad o transdisciplinariedad y actúan, al mismo tiempo, como significados oponentes de nociones como ciencia tradicional, disciplina o paradigma clásico. Si bien han sido analizadas desde la Filosofía y desde las Ciencias Sociales las conexiones entre los nuevos modelos para representar el conocimiento científico y los modelos de representación del funcionamiento de la sociedad posmoderna, en los documentos analizados suelen equipararse (a veces hasta confundirse) la alusión a la complejidad de la sociedad globalizada actual y la referencia a la idea de complejidad para indicar nuevas perspectivas epistemológicas de la ciencia. La primera señalaría un uso más bien general del término, de tipo sociológico pero sin rigor teórico, mientras que la segunda apelaría al sentido epistemológico 
estricto de dicha noción. Asimismo, estas diferenciaciones no siempre son reconocidas o los significantes usados no motivan en todos los casos esclarecimientos conceptuales, pasando a conformar, entonces, constructos pedagógicos que operan, circulan y ordenan los discursos de forma poco rigurosa. Asimismo, llama la atención el mero papel adaptativo que le cabe a la FD, y que el artículo propone, en relación a los procesos de globalización contemporáneos.

Por su parte, en pronunciamientos provenientes de organismos estatales, también encontramos referencia a la necesidad de un cambio en la visión de la ciencia que sustentan los profesorados de Matemática y Ciencias Naturales. En su informe final, que contiene recomendaciones para la elaboración de políticas educativas, la Comisión Nacional para el Mejoramiento de la Enseñanza de las Ciencias Naturales y la Matemática ${ }^{3}$ insiste en la necesidad de una transformación de la enseñanza de la ciencia y de la FD, en las cuales diagnostica la prevaleciente presencia de visiones tradicionales: "La educación tradicional en el aula ignora casi por completo el proceso de generación de las ideas, enfocando su atención casi exclusivamente en el producto final de la ciencia..." (ARGENTINA, 2007a, p. 10). En otro apartado se afirma:

[...] será necesario que la formación permita superar las visiones simplistas de la ciencia y del trabajo científico, así como las visiones que hacen del conocimiento científico algo extremadamente difícil y naturalizan el fracaso escolar. Ahora bien, para superar estas visiones así como aquellas prácticas pedagógicas tradicionales mencionadas en los apartados anteriores, es preciso que las propuestas de renovación sean vividas, vistas en acto. Sólo así resulta posible que las mismas tengan efectividad y que los futuros docentes (o los que ya están activos) rompan con la visión unilateral de la docencia recibida hasta el momento... (ARGENTINA, 2007a, p. 10)

En este documento advertimos que la superación de las visiones simplistas de la ciencia se vincula a tres premisas. En primer lugar, el cambio en la enseñanza tiene una dimensión política, pues debe contribuir a una alfabetización científica de los sujetos como parte de su formación ciudadana. En segundo término, dicha superación debe lograrse por propuestas de enseñanza de la ciencia que enfaticen los procesos de producción de los conceptos, sugerencia que se apoya en resultados de investigación actuales de las corrientes cognitivistas sobre adquisición de conceptos científicos. Por último, se asume que sólo habrá transformación en la medida en que las visiones complejas de la ciencia aniden en prácticas concretas de aprendizaje de los futuros profesores.

La característica saliente es que el armazón discursivo de la Comisión Nacional de expertos se conforma polarizando dos modos de entender la enseñanza de la ciencia: una "tradicional”, caracterizada por un cúmulo de contenidos canónicos dispensados por el profesor a

\footnotetext{
${ }^{3}$ La Comisión Nacional para el Mejoramiento de la Enseñanza de las Ciencias Naturales y la Matemática fue conformada por expertos de las áreas disciplinares y por funcionarios del Ministerio de Educación de la Nación Argentina.
} 
alumnos cuya mente estaría "en blanco"; y "un enfoque actual” que reconoce las preconcepciones que los estudiantes traen al aula, en relación a las cuales construirán los nuevos conocimientos de acuerdo a la actividad de un docente que ahora sería más bien un guía o un facilitador. En este sentido, la Comisión propone instalar en la FD y, por ende, en las escuelas, metodologías de aprendizaje que repliquen aspectos de lo que un científico haría en su actividad cotidiana de investigación, a saber:

[...] la experimentación, las preguntas frecuentes, el diálogo socrático, los razonamientos rigurosos y carentes de circularidades, el poder compartir y argumentar las propias ideas, cuestionarlas, generar actividades de abstracción, trabajo en equipo, formulación de hipótesis, observaciones y deducciones, generación de modelos. (ARGENTINA, 2007a, p. 11-12).

En cuanto a las propuestas concretas para mejorar la FD, los artículos sobre Didáctica Específica como el mencionado anteriormente y el informe de la Comisión Nacional, coincidirían en líneas generales en:

- Actualizar los contenidos disciplinares y dar centralidad a la Didáctica Específica;

- Orientar los procesos de aprendizaje de los futuros profesores hacia razonamientos de tipo complejo;

- Que el futuro docente sea el centro del aprendizaje;

- Comprender conceptos científicos en secuencias no lineales, en tramas que muestren su devenir y sus relaciones;

- Priorizar la formación en contextos experimentales a partir de la resolución de problemas;

- Fortalecer los Institutos transformándolos en centros de investigación, mejorando los equipamientos y recursos existentes, financiando proyectos de mejora.

A diferencia de las perspectivas que adoptan la Complejidad e instan a revisar la organización disciplinar de los conocimientos, los últimos Lineamientos Curriculares nacionales para la FD elaborados desde el organismo encargado de las políticas de formación en Argentina, toman recaudos con respecto a la organización multi o interdisciplinar de los planes de estudio de los Profesorados, sugiriendo más bien currículos basados en la disciplina de base para la que se forme: "En los profesorados de educación secundaria (se debe) preservar la formación específica en la disciplina particular objeto de la formación y sus contenidos derivados, evitando la organización en pluri-disciplinas o inter-disciplinas, en especial en los primeros años de estudio" (ARGENTINA, 2007b, p. 37).

Se destaca también en este documento la sugerencia de que sea en la Didáctica Especial del último año de las carreras donde se abran espacios de experimentación e innovación de la enseñanza, apelando al conocimiento del estado actual del campo investigativo en la enseñanza de las ciencias naturales.

Entre los planteos que vienen de la mano del pensamiento complejo (los cuales instan a revisar la organización disciplinar del saber) y la insistencia en la organización disciplinar que recomiendan los Lineamientos parece existir una contradicción, si es que advertimos que las revisiones de los currículos de FD pretenden orientarse hacia perspectivas complejas del conocimiento. No obstante, es posible interpretar este contrasentido comprendiendo que los 
saberes de los ámbitos epistemológicos y disciplinares son reelaborados y reinterpretados en virtud de tramas históricas - políticas - epistémicas propias del campo de la enseñanza. En realidad, la historia de la escolarización de las ciencias en nuestro país pone en evidencia la fuerte presencia de los demarcamientos disciplinares y de las perspectivas profesionalizadas de las ciencias ("ciencias de laboratorio") como formato del saber que ha ingresado desde principios del siglo XX en el nicho escolar (AISENSTEIN, 2000; GOODSON, 1995). Estas posturas han sido ampliamente defendidas y legitimadas por instituciones como las universidades, las asociaciones de profesores y el Estado. A partir de ello podemos comprender cómo se ha ido configurando históricamente la formación de profesores para los niveles medio y superior, cuyos posicionamientos continúan arraigados dado que se encuentran inscriptos en luchas materiales y simbólicas por el reconocimiento de los sujetos que sustentan dichas posiciones. Por otro lado, si bien las propuestas de los Institutos Superiores y de algunas Universidades argentinos suelen centrarse en la formación de profesores para las Ciencias Naturales, continúa operando la idea de que una formación sólida requiere la profundización en la disciplina de base.

En otro sentido, principios como los de flexibilidad y apertura intentan ser recuperados pero sólo para proponer una cierta organización institucional de los Profesorados que sea acorde a lo que se interpreta como nuevos desafios:

EL PLAN NACIONAL DE FORMACIÓN DOCENTE, en el que se inscribe la línea general de trabajo del PROYECTO DE MEJORA INSTITUCIONAL, apunta al desarrollo de instituciones dinámicas y abiertas, que logren constituirse y consolidarse como ambientes de formación y aprendizaje articulados en redes sociales, educativas y académicas, en concordancia con los actuales requerimientos pedagógicos y organizacionales. (ARGENTINA, 2008, p. 3, énfasis del autor)

Los organismos nacionales encargados de las políticas de FD proponen, así como lo hace también la recomendación de la Comisión, asignar recursos materiales y económicos con el fin de que los Institutos Superiores de Profesorado que dictan carreras en Matemática o Ciencias Naturales renueven contenidos y prácticas de enseñanza a partir de generar capacidad de intercambio de conocimientos, experiencias, recursos y proyectos al interior de la propia institución y con organizaciones externas. Se consolidan, así, en políticas oficiales y diseños institucionales nociones como las de red, organizaciones flexibles, sistemas abiertos, entre otras, que supondrían jaquear - o al menos poner en entredicho - la noción misma de disciplina; esto si suponemos que toda transformación en las prácticas sociales de producción, reproducción, circulación y distribución de saberes produce impactos en las lógicas disciplinares, por tanto en la epistemología basada en la disciplina. Sin embargo, estos procesos no son advertidos ni señalados por los documentos oficiales que hablan de la transformación de la FD en Ciencias. 


\section{La perspectiva de la Complejidad en planes de estudio y programas de cátedra}

Si pasamos ahora al contenido de algunas propuestas de FD en Física de Instituciones de Formación Docente ${ }^{4}$, advertimos que se menciona la necesidad de formar profesores desde una mirada compleja y se destaca la importancia de incluir la Epistemología en los planes de estudio como campo disciplinar que viabiliza comprender las perspectivas clásicas y contemporáneas en el desarrollo de la Física. Por otro lado, la Didáctica Especial se transforma, en diseños como estos, en disciplina articuladora de toda la propuesta de formación, lo cual se enuncia como innovación respecto de planes "tradicionales". Es la Didáctica Específica la que debe poner en relación, según estas propuestas, los desarrollos de la Física y las Ciencias Naturales con estrategias complejas e innovadoras de enseñanza, acordes a las situaciones escolares contemporáneas y a las nuevas perspectivas epistemológicas sobre la ciencia. En este sentido, también se valora el ingreso de la Investigación Educativa como asignatura desde la que se pueden organizar ciertas asignaturas (como la Didáctica Específica) por cuanto daría la posibilidad de visualizar el carácter abierto, conjetural, constantemente revisado del conocimiento en materia de enseñanza de la ciencia.

Sin embargo, cuando nos sumergimos en un análisis minucioso de los espacios físicos y simbólicos que dichas asignaturas adquieren en la lógica general de los planes de estudio, así como de los programas de cátedra ${ }^{5}$ encontramos un panorama mucho más heterogéneo y paradójico de lo que el discurso compacto del plan o del diseño muestra. Más allá de los pronunciamientos e intenciones manifiestas en los diseños y fundamentaciones de planes de estudio, se torna metodológicamente sustancial en este tipo de abordaje echar un vistazo a la organización efectiva de las materias y a sus contenidos, tarea que arroja interesantes puntos de interrogación.

Otros trabajos relacionados con este tema muestran que la disposición de saberes de la FD suele contemplar una repartición de funciones o una división intelectual del trabajo en la que, por ejemplo, la Didáctica funciona como dadora de estrategias para enseñar contenidos que ya vendrían proporcionados por las materias de la formación disciplinar, es decir, por las materias de Física y afines (LUNA; CASTELLS; GIORGI, 2010). Esta repartición epistémica queda develada por cuanto la asignatura de Didáctica Específica no contiene generalmente elementos conceptuales en la mayoría de los programas analizados, organizando su propuesta en torno a unidades empíricas tales como "selección, planificación y evaluación de contenidos de Física", "la Física en el nivel secundario", "la construcción de estrategias innovadoras de enseñanza", o "el diseño curricular de nivel medio para la Física". Algunos programas, además, incorporan elementos de la corriente de la Didáctica por modelos, que como línea investigativa ha

\footnotetext{
${ }^{4}$ Las conclusiones de este apartado se basan en el análisis de cinco planes de estudio y programas de Profesorados universitarios y no universitarios de Física en vigencia de diversas provincias de Argentina. Por razones de índole metodológica y ética se resguarda la identidad de las instituciones.

${ }^{5} \mathrm{La}$ indagación de lógicas discursivas y epistémicas presentes en planes y programas no implica reconocer para las prácticas efectivas de formación idénticas lógicas, creyendo que la enseñanza institucional cotidiana se ajusta a la letra de los documentos. Sí podemos sostener que los documentos enuncian ciertos modos de visibilizar la formación que son producto de prácticas sociales específicas con el saber.
} 
elaborado una clasificatoria de modelos de enseñanza según la concepción de ciencia y de sujeto de aprendizaje que sustenten los mismos. De este modo, la Didáctica en la FD se atribuiría ser el ámbito de saber que proporciona modelos de enseñanza dentro de un menú de posibles opciones que el futuro profesor deberá evaluar - según lo afirman varios programas - de acuerdo al contexto de enseñanza, el tipo de contenido y de alumno con el que se encuentre, pues en estos planteos la enseñanza siempre es situada como instancia externa a la propia formación, en instituciones y aulas que nunca son las del propio Profesorado.

\section{La formación docente como dispositivo pedagógico}

En la lectura del corpus, la exploración de su entramado discursivo suscitó el siguiente interrogante: ¿qué episteme está organizando la noción de cambio o de transformación allí presente? Como conclusión general, que a la vez se convierte en hipótesis para futuras indagaciones, podemos decir que los discursos conciben la FD como un espacio depositario de las nuevas perspectivas epistemológicas de las Ciencias, situando allí la posibilidad de actualización de las prácticas de enseñanza y aprendizaje. Este posicionamiento conlleva una serie de interpretaciones sobre la formación de los profesores - y sobre la enseñanza en general - cuyos efectos epistémicos y políticos es preciso desentrañar.

En primer término, cuando se polarizan opciones tales como lo "tradicional/simple" versus lo "actual/complejo" en materia de FD y cuando las prácticas vigentes o arraigadas se significan como "lo distorsionado", se revela una lógica que mucho debe al paradigma clásico del conocimiento, aunque esto sea algo invisible para las pretensiones del propio discurso. Estos discursos educativos aún conciben de forma binaria las prácticas de conocimiento, reavivando así la búsqueda de una refundación siempre radical de las prácticas. En este contexto, las condiciones existentes se vuelven dicotómicas y excluyentes, puesto que donde hay error no podría haber saber y habría que fundar el "edificio" desde los "cimientos", metáfora cartesiana que bien puede ilustrar los modos de relación con el saber que operan en estos discursos. Este tipo de concepción de la formación y de la enseñanza juzga que los planes de estudio y las prácticas institucionales sólo requieren "actualizaciones", posibles de concretar si se cambia un modelo por otro o un paradigma por otro. Sin embargo, creemos que la lógica dicotómica de los planteos contribuye a oscurecer las tramas por medio de las cuales las instituciones y los sujetos han forjado su existencia y sus prácticas.

Cuando hablamos de "tramas" nos referimos a dimensiones interrelacionadas que juegan delineando las propuestas de formación y que generalmente no son conscientes para sus protagonistas porque, como dice Bourdieu (1991), se hacen "cuerpo". Las tradiciones hegemónicas en los campos pedagógico y científico, las estructuras e historias institucionales, las trayectorias de los cuerpos docentes, la configuración histórica del sujeto docente de nivel medio y superior en Argentina, las lógicas de las políticas públicas, las lecturas que las instituciones hacen de la realidad y de los campos disciplinares, el tipo de relación establecida con el nivel educativo para el que se forma, los modos en que se han conformado las disciplinas escolares (como ser el caso de la Física y de las Ciencias Naturales en Argentina), son dimensiones que operan mediando aquello que la FD contextualizará y recontextualizará como saberes legítimos a ser transmitidos. 
De acuerdo a lo expuesto, se hace necesario recurrir a nociones y categorías que permitan profundizar y comprender ese rol de mediación y creación de conocimientos que ejerce todo espacio de formación de docentes respecto de lo que acontece en los campos académicos y disciplinares, así como en la esfera social y estatal. Sostenemos que un punto clave en este enfoque radica en el modo de concebir la conformación de los conocimientos de la formación, asunto abordado a continuación.

Los saberes que forman parte de una propuesta de formación surgen de procesos histórico-sociales de creación y recreación de conocimientos que se dan, en parte, en las propias instituciones formadoras, en cuyas prácticas se reinterpreta lo que acontece en espacialidades más amplias (políticas estatales, sistema educativo, mercado laboral, campo editorial, etc.). Creemos que teorizar o construir propuestas sobre formación de profesores requiere historizar los saberes que la conforman y las estructuras institucionales y epistémicas que las sustentan. Este posicionamiento reconoce como figuras teóricas de relevancia a Yves Chevallard (1991), quien propone un análisis antropológico de los saberes, a Basil Bernstein (1974, 1990, 1993, 1998), que formula un análisis sociológico del conocimiento transmitido por el espacio escolar y a Michel Foucault (1972) con sus aportes para un análisis arqueológico de los saberes. Si bien reconocemos búsquedas teóricas distintas entre ellos, creemos que sus planteos posibilitan poner en perspectiva histórica la producción de los conocimientos que forman parte de la formación y detener la atención en las condiciones de emergencia y disposición de los saberes. En esta oportunidad aludiremos a ciertos aportes provenientes de la conceptualización que hace Bernstein sobre el dispositivo escolar, dado que nos acerca a la noción de Formación Docente que intentamos sostener.

Para ese autor, y debido a los complejos procesos de división del trabajo que caracteriza a nuestras sociedades capitalistas, el espacio escolar es un terreno de "mediación" entre los conocimientos que pretende transmitir la sociedad y los individuos que deben adquirirlos. La clave teórica de Bernstein fue haber construido conceptos que dilucidan los mecanismos y regulaciones que rigen la producción de conocimientos en tal espacio, lo cual fue posible luego de décadas de investigación en escuelas inglesas. Con ello el autor quiso demostrar que las instituciones educativas no sólo transmiten conocimientos, sino que los producen/reproducen.

Tal como lo hace el dispositivo lingüístico, el dispositivo pedagógico selecciona y regula las combinaciones de aquello que es dicho, por lo tanto, no es sólo un medio neutral que "transporta" comunicaciones o mensajes. Bernstein (1998) define al dispositivo pedagógico como un sistema de producción de mensajes con reglas internas relativamente estables que regula las comunicaciones legítimas entre enseñantes y adquirientes. Esos mensajes actúan de forma selectiva sobre el potencial de significado (todos aquellos significados susceptibles de entrar al espacio escolar o pedagógico) reforzando algunas realizaciones y restringiendo otras. Las reglas del dispositivo son:

- distributivas: definen qué es lo transmisible, el límite entre lo que es pensable e impensable en una sociedad y tiempo dados, por tanto, operan haciendo una distinción entre dos tipos de conocimientos (pensable e impensable). Por ejemplo, nuestra cultura escolar considera a lo esotérico como conocimiento impensable o no transmisible.

- recontextualizadoras: tienen la función de apropiarse de otros discursos, como los del campo de las disciplinas científicas, y dislocarlos para recolocarlos en otra situación y con- 
texto, en vistas a su transmisión. Estas reglas tienen por función seleccionar y crear los temas pedagógicos especializados de acuerdo al contexto escolar.

- evaluadoras: establecen la configuración de tiempos, espacios y textos que permite concretar la práctica pedagógica; allí los sujetos nos socializamos y constituimos nuestra conciencia, en gran medida, de acuerdo con lo que es "pensable", aunque el autor no desconoce que el espacio escolar es terreno de luchas por el control y transformación de los significados que allí circulan.

Las operaciones de recontextualización de conocimientos implican, entonces, procesos de "creación" de conocimientos por y para el espacio de la formación en los que se define aquello que será transformado en contenido y cómo eso debería ser transmitido. Esas operaciones son ejercidas por diversos agentes y campos, entre los que Bernstein (1998) distingue el campo recontextualizador oficial (el Estado con sus ministerios y funcionarios) y el campo recontextualizador pedagógico (compuesto por las instituciones formadoras, las universidades, editoriales, etc.). Con ello el autor nos advierte que la selección, jerarquización, tematización de los conocimientos escolares pasan por distintos "filtros" que construyen y reconstruyen aquello que será establecido como saber a transmitir. La existencia de estos campos y agentes diferenciados hace que exista una relativa autonomía y tensiones entre uno y otro.

Parece que las últimas políticas públicas en Argentina intentan debilitar la separación entre los agentes recontextualizadores oficiales y los agentes que trabajan en la producción del discurso de las disciplinas (académicos, investigadores), promoviendo que sean comisiones o grupos de expertos los que definan criterios y contenidos de Ciencias Naturales, tanto para la escolaridad básica y media, como para la formación de profesores. Estos movimientos, cuyo efecto imaginado sería el pasaje de un flujo de saberes actualizados hacia la escuela y los futuros docentes, podrían no tener el resultado pretendido en la medida en que se desconocen las peculiaridades de la formación como campo recontextualizador inscripto en una historia, una estructura institucional y unas trayectorias específicas de los sujetos que las transitan. En la misma línea, en otro trabajo indagamos cómo la organización de conocimientos de distintos planes de estudio de Profesorados de Física en Argentina tiene vinculación con múltiples aspectos institucionales y sociales, lo cual produce propuestas de formación heterogéneas (LUNA; CONCARI; CASTELLS, 2009). Las características institucionales de los profesorados (si tienen o no tradición universitaria), el grado de autonomía respecto del Estado, la historia de surgimiento de cada propuesta de formación, las lecturas que los sujetos hacen de los contextos de trabajo de los futuros profesores, la trayectoria de los formadores, fueron dimensiones consideradas relevantes para las investigadoras en tanto les permitieron comprender las diferencias curriculares entre los planes de estudio.

Este espacio recontextualizador o productor de saberes, la FD, no suele ser reconocido por los discursos analizados, los cuales más bien se limitan a concebirla como un lugar receptivo de conocimientos (los contenidos del "Paradigma de la complejidad"), en este caso desde el campo epistemológico al pedagógico. Estas orientaciones que asumen los discursos sobre FD, junto a las epistemes que los estructuran, no dejan de señalarnos la presencia - aún prioritaria - de una noción idealista del conocimiento, como si éste y los modos de su producción no encarnaran en cuerpos, instituciones, reglamentos, modos de reconocimiento entre sujetos y saberes, y se reelaboraran desde allí para darle ciertos y nuevos destinos. Más aún, las propuestas de revisión de la FD analizadas, parecen omitir el análisis de las condiciones de emergencia, 
circulación, producción y reproducción de los saberes en la formación y de la formación. Así, por ejemplo hablar de distorsión (que equivale a hablar de saberes equivocados o anticuados que los docentes deberían desterrar y cambiar) es una posición que nos impide poder visibilizar las inscripciones particulares de los saberes en unas tramas históricas, políticas e institucionales que les dieron y dan sentido. En este contexto, vemos reeditarse en discursos contemporáneos que bregan por la instalación del Paradigma de la Complejidad en la educación, huellas de una perspectiva ilustrada del conocimiento que desencadena - aunque intente evitarlo - políticas de formación que metaforizan una cruzada contra lo que califican que sería una mera obsolescencia de los conocimientos.

\section{Hacia una resignificación de la noción de "complejidad" en la formación de profesores}

A partir del análisis de la lógica que organiza los discursos del corpus y de los aportes teóricos recuperados planteamos otra noción de complejidad para pensar la formación de profesores de ciencias. Así, una mirada "compleja” sobre la FD debería permitir interrogar las contradicciones aparentes entre discursos y prácticas o entre diversos discursos, tal como vimos que se producía entre las recomendaciones de expertos y los lineamientos curriculares respecto de la organización interdisciplinar o disciplinar de la formación. Allí pudimos vislumbrar el encuentro de posturas divergentes en la medida en que ésta se torna un espacio de disputas profesionales, institucionales y epistemológicas de acuerdo a la historicidad que atraviesa a los profesores, las instituciones de formación y la conformación de los campos de conocimiento de la formación. Entrever estos procesos nos aleja de la interpretación de la formación como mero ambiente inocuo de traspaso de conocimientos desde el campo académico y científico hasta los futuros profesores.

Por su parte, los discursos institucionales presentes en planes y programas también muestran cierta persistencia de una "epistemología" institucional que se cree superada. Cuando hablamos de "epistemología institucional" queremos referirnos a modos concretos de relación con los saberes y los campos disciplinares que tienen las instituciones y sus sujetos. En ese sentido, en relación a la organización curricular detectamos una fuerte permanencia de lógicas epistémicas objetivistas, con cierto realismo ingenuo, en tanto lo real (identificado con la formación disciplinar o con las aulas de las escuelas de inserción futura de los estudiantes de profesorado) continúa concibiéndose como algo externo, independiente de la mirada y la posición del sujeto (NAJMANOVICH, 2008; VON GLASERSFELD, 1994), premisa que justamente el paradigma de la Complejidad puso en crisis. Como hemos intentado mostrar, este objetivismo se devela cuando el cúmulo de materias de la formación disciplinar se ven - y son vistas por las demás - como los contenidos inconmovibles, con una estructuración fija y a priori que debe ser transmitida por el futuro profesor y a la que sólo restaría “añadir” buenas metodologías y recursos de enseñanza (rol que suele ser otorgado a la Didáctica). Al estar la Física, o cualquier otra disciplina, situada en el lugar de lo que ya está dado y su propia estructura no se interroga, se renueva cierta estética representacionalista (NAJMANOVICH, 2008) o la relación objetivista en la que a la Didáctica sólo le queda ajustar su función tecnológica (BERNSTEIN, 1993). Este posicionamiento impide comprender que los contenidos de enseñanza de Física también son una construcción compleja en la que han intervenido instancias estatales, académicas, ins- 
titucionales, profesionales y que se ha dado en medio de juegos de poder y reconocimientos históricamente situados.

Además, si la Didáctica Especial, la Epistemología o la Investigación Educativa sólo ingresan a los planes como asignaturas o temas, pero no son territorios de pensamiento capaces de potenciar institucionalmente espacios de interrogación y reconocimiento de las lógicas epistémicas presentes en las disciplinas y en la formación, aquellas prácticas y conocimientos juzgados como obsoletos o tradicionales probablemente sigan persistiendo a pesar de la existencia de una voluntad conciente de cambios.

Si la FD se concibe sólo como un cúmulo de materias o como un espacio neutro y depositario de lo que acontece en otros campos y no logra convertirse en zona crítica de múltiples reconocimientos (de las lógicas epistémicas, de los juegos de poder, etc.), el aula o la propia formación seguirán siendo un no lugar (LIZCANO, 2006), y sus discursos seguirán contribuyendo a instituir la certeza de que todos, en cualquier tiempo y espacio podríamos aprender lo mismo y de igual modo. Los conocimientos se vuelven, así, entidades abstractas, desarraigadas de los sujetos y las prácticas que les dieron vida, transformándose en pura información (LIZCANO, 2006), al tiempo que en las políticas del saber que atraviesan a las instituciones se configura la ilusión de poder sustituir un modelo por otro, un paradigma por otro.

Por último, se vuelve necesario a nuestro criterio que toda revisión epistemológica al interior de la formación de profesores sitúe históricamente el advenimiento del pensamiento complejo, que tampoco supone una corriente teórica homogénea posible de ser trasladada a la enseñanza. Este análisis se torna sustancial, dado que los Profesorados para nivel secundario en Argentina se organizaron históricamente alrededor del lenguaje disciplinar, por lo que los modos de relación con el conocimiento de los sujetos que a ellos pertenecen, las estructuras curriculares, la organización departamental de las instituciones, los reglamentos, los sistemas de correlatividades, etc., responden en gran parte a la lógica de la disciplina. En todo caso, la recuperación de los aportes de las epistemologías contemporáneas en la FD debería ir de la mano de una revisión de las crisis y transformaciones acontecidas en las disciplinas, sobre todo en las Naturales y Experimentales, pues los nuevos planteos son comprensibles en el marco de lo que la organización disciplinar del saber dejó como preguntas abiertas y problemas irresueltos.

Otros efectos podrían surgir y otros caminos lograrían abrirse si el paradigma de la Complejidad no fuera leído como un precepto externo de cómo deben delinearse ahora las prácticas de enseñanza, en tanto el mandato que - parece - debiera cumplirse sería la "actualización" de los profesorados, como si tal cosa pudiera concretarse descontextualizando la formación de las apropiaciones efectivas de conocimientos que hacen las instituciones y los sujetos. La recuperación de los aportes de la perspectiva de la Complejidad más bien podría tener un rol heurístico antes que prescriptivo, en la medida en que pudiese favorecer el esclarecimiento y reconocimiento de la lógica que ha forjado históricamente la estructura curricular e institucional de los profesorados de nivel medio y superior en Argentina: la disciplina. Con ello las instituciones formadoras tendrían la posibilidad de ejercer una vigilancia en torno a la constitución histórica y epistemológica de la formación, lo cual podría generar mayor autonomía en la definición de las políticas recontextualizadoras de sus propios saberes. 
El paradigma de la complejidad ...

\section{Agradecimientos}

Este trabajo ha sido realizado en el marco del proyecto de investigación PICT 2006 1427:

"Caracterización de la Formación Docente en Física en Argentina", de la Universidad Nacional del Litoral, Argentina.

\section{Referencias}

ACOSTA, R. Formación de profesores en el paradigma de la complejidad. Educación y

Educadores, Chía, v. 9, n. 1, p. 149-157, 2009. Disponible en: <http://educacionyeducadores. unisabana.edu.co/index.php/eye/article/view/653/738>. Visitado el: 23 jul. 2014.

AISENSTEIN, A. Las ciencias exactas y naturales en la escuela: una mirada desde el currículum (1870-1983). In: GVIRTZ, S. (Dir.). El color de lo incoloro. Buenos Aires: Novedades Educativas, 2000. p. 23-57.

ARGENTINA. Ministerio de Educación, Ciencia y Tecnologia. Comisión nacional para el mejoramiento de la enseñanza de las ciencias naturales y la matemática: informe final. Buenos Aires, 2007a.

. Ministerio de Educación. Instituto Nacional de Formación Docente. Lineamientos curriculares nacionales para la formación docente inicial. Buenos Aires, 2007b.

. Ministerio de Educación. Instituto Nacional de Formación Docente. Plan nacional de formación docente. Buenos Aires, 2007c.

- Ministerio de Educación de la Nación. Instituto Nacional de Formación Docente. Proyecto de mejora institucional para la formación docente en ciencias naturales y matemática. Buenos Aires, 2008.

BERSNTEIN, B. Clasificación y enmarcación del conocimiento educativo. In: Class, codes and control. London: Routledge, 1974. v. 1. (Traducido con permiso del autor por Mario Díaz).

La estructura del discurso pedagógico. Madrid: Morata, 1993.

Pedagogía, control simbólico e identidad. Madrid: Morata, 1998.

Poder, educación y conciencia. Barcelona: El Roure, 1990.

BOURDIEU, P. El sentido práctico. Madrid: Taurus, 1991.

CHEVALLARD, I. La transposición didáctica: del saber sabio al saber enseñado. Buenos Aires: Aique, 1991.

FOUCAULT, M. La arqueología del saber. México: Siglo XXI, 1972.

GOODSON, I. Historia del currículum: la construcción social de las disciplinas escolares. Barcelona: Pomares: Corredor, 1995.

LIZCANO, E. Metáforas que nos piensan: sobre ciencia, democracia y otras poderosas ficciones. [Madrid]: Bajo Cero: Traficantes de Sueños, 2006.

LUNA, M. V.; CASTELLS, M. C.; GIORGI, S. Profesorados de física: un análisis de la didáctica específica desde las regulaciones internas de su discurso. In: CONGRESO INTERNACIONAL DE DIDÁCTICAS ESPECÍFICAS PODER, DISCIPLINAMIENTO Y EVALUACIÓN DE SABERES, 2., 2010, Buenos Aires. Actas... Buenos Aires: UNSAM, 2010. 
Luna, M. V.

LUNA, M. V.; CONCARI, S.; CASTELLS, M. Una mirada a la formación docente universitaria en física desde planes de estudio. In: JORNADAS DE FORMACIÓN DOCENTE UNIVERSITARIA, 2009, Rosario. Actas... Rosario: Escuela de Ciencias de la Educación: Facultad de Humanidades y Artes, 2009.

NAJMANOVICH, D. E1 lenguaje de los vínculos: de la independencia absoluta a la autonomía relativa. Buenos Aires: Paidós, 1995.

Mirar con nuevos ojos: nuevos paradigmas en la ciencia y pensamiento complejo. Buenos Aires: Biblos, 2008.

POPKEWITZ, T. Sociología política de las reformas educativas. Madrid: Morata, 1994.

VON GLASERSFELD, E. Despedida de la objetividad. In: WATZLAWICK, P.; KRIEG, P. (Comp.). El ojo del observador: contribuciones al constructivismo. Barcelona: Gedisa, 1994. p. 19-31.

Disponible en: <http://docencia.izt.uam.mx/egt/Cursos/MetodologiaMaestria/Glasersfeld.pdf>. Visitado el: 23 jul. 2014. 